Cahiers $d u$ MONDE RUSSE

\section{Cahiers du monde russe}

Russie - Empire russe - Union soviétique et États indépendants

$50 / 1 \mid 2009$

Écrits personnels. Russie XVIII $-\mathrm{XX}^{\mathrm{e}}$ siècles

\title{
Quand la terreur se mêle de narration
}

Le cas d'un journal sans nom (1936-1937)

\section{Véronique Garros-Castaing}

\section{(2) OpenEdition}

1 Journals

Édition électronique

URL : https://journals.openedition.org/monderusse/9156

DOI : 10.4000/monderusse. 9156

ISSN : $1777-5388$

Éditeur

Éditions de l'EHESS

Édition imprimée

Date de publication : 31 mars 2009

Pagination : $125-138$

ISBN : 978-2-7132-2259-7

ISSN : $1252-6576$

Référence électronique

Véronique Garros-Castaing, "Quand la terreur se mêle de narration », Cahiers du monde russe [En ligne], 50/1 | 2009, mis en ligne le 01 janvier 2009, consulté le 03 septembre 2022. URL : http:// journals.openedition.org/monderusse/9156; DOI : https://doi.org/10.4000/monderusse.9156 


\section{QUAND LA TERREUR SE MÊLE DE NARRATION}

\section{Le cas d'un journal sans nom (1936-1937)}

Lecteur intrus : l'histoire posthume de ce « journal sans nom » nous place singulièrement, on va le voir, dans cette posture à laquelle serait censée nous soumettre tout journal dit intime. Et, du fait de cette « intrusion », son auteur restera anonyme, N. en l'occurrence ${ }^{1}$. En novembre 1937, quelques jours avant d'être arrêté puis fusillé, N. confie à sa fille de dix-huit ans ses Cahiers - afin de les «mettre à l'abri ». Celle-ci a tenu scrupuleusement cette promesse jusqu'en $1991^{2}$ pour pouvoir, enfin, l'ébruiter, lui donner vie. Des différends familiaux sur l'opportunité de les publier ont eu (temporairement ?) raison de ce désir.

Aussi doit-on se contenter pour l'heure d'esquisser, en guise d'une biographie détaillée, la silhouette de N. Celui-ci occupe une haute fonction au sein d'un Commissariat du peuple; il a une cinquantaine d'années lorsqu'il inaugure son journal le 1er janvier 1936, s'exilant, sans le savoir encore, en écriture. Une expression, aussi laconique que ces détails biographiques, pourrait le caractériser alors : il est, peut-être comme de nombreux vieux bolcheviks (mais un vieux bolchevik qui, s'il a côtoyé de nombreux opposants de renom, n'a pour autant jamais rejoint leur mouvement ou leur mouvance), un homme-frontière, un homme de ou en transit. Un homme qui, en l'occurrence, n'avait politiquement et statistiquement guère de chance d'échapper au sort que l'on sait.

\footnotetext{
1. N. plutôt que X. car le russe adjoint à cette notion d'anonymat un supplément de sens.

2. Date à laquelle elle m'a remis ces Cahiers alors que j'avais entrepris une recherche sur les journaux personnels en période de terreur, dans le cadre d'un projet plus global dirigé par Marc Ferro. Cette recherche a donné lieu à un ouvrage collectif: Véronique Garros, Natalia Korenevskaya, Thomas Lahusen, eds, Intimacy and Terror : Soviet Diaries of the 1930s, New York: The New Press, 1995 ; version allemande publiée sous le titre Das wahre Leben : Tagebücher aus der Stalin-Zeit, Berlin : Rowohlt Berlin, 1998.
} 
Ce journal se fait l'écho d'une terreur innommable, d'une situation « surréaliste » (au sens où l'on ne sait plus dans quelle réalité l'on vit ${ }^{3}$ ), d'un monde où la mort - pire : la disparition - rôde nuitamment en un silence assourdissant. À l'inverse de la terreur « Rouge », celle-ci, la « Grande » ne veut pas d'exécution publique, de corps suppliciés exposés, de sang qui coule aux yeux de tous : bizarrement, elle rend la mort taboue ${ }^{4}$. Elle lui préfère une mise en scène visant à neutraliser (voire esthétiser) son essence : en ce sens, les spectaculaires procès de Moscou peuvent être considérés comme moments de pure négation de la terreur ${ }^{5}$. Or, on le sait historiquement, une terreur sans nom redouble de violence : ne pas afficher cette dernière, ou feindre de l'ignorer, c'est en changer la nature et bouleverser, en conséquence, les comportements tant sociaux qu'individuels. Le journal de N., comme tant d'autres, fait état de cette violence supplémentaire qu'ont constituée le non-dit et la difficile, voire impossible, intelligibilité de la réalité environnante dès lors qu'elle ne peut être qualifiée, nommée. En ce cas, quelle stratégie, quelle posture morale et/ou politique adopter quand le monde alentour ne peut être l'objet que de lancinantes interrogations sans réponses? Dans le journal de N., cette terreur sans nom se présente sous différentes formulations qui renvoient toutes à un « innommable », précisément :

Je ne sais vraiment pas où les gens ont la tête. [Les « gens » en l'occurrence sont ceux qui l'accusent de diffamer Stalin en raison d'une conversation qu'il a eue avec ce dernier à propos de Smilga, désormais démasqué comme ennemi du peuple - V.G.]

Le diable seul sait ce qu'il se passe ! [Conversation avec un ami]

Pourquoi ? [...] Je ne peux qu'essayer de deviner, mais sur le fond personne, pour l'instant, ne sait rien. Personne ne comprend ce qui se passe et ce qui s'est passé dans les hautes sphères ; d'où proviennent et où se sont nichés les ennemis du peuple.

Où est la réalité, la vérité, la justice ?

Cette forte demande sociale a sans doute résonné, au sein d'un silence assourdissant comme nous l'avons dit, dans l'esprit d'innombrables Soviétiques. Souvenons-

3. Nous empruntons cet emploi du terme «surréaliste » à Alain Cottereau, défini lors d'une intervention au cours du colloque «Expériences limites, ruptures et mémoires. Dialogues avec l'Amérique latine », Paris, 18-20 octobre 2006, Centre d'études des mouvements sociaux, Institut Marcel Mauss, EHESS

4. Bizarrement, car l'URSS ne faisant pas partie alors des pays « avancés » dont parle Georges Auclair ; on pourrait malgré tout lui appliquer sa réflexion : « [...] survivance d'un rite de purification collective, elle est devenue un spectacle interdit ou, si l'on veut, tabou - , un spectacle dont émane un mana impur » (Le Mana quotidien : Structures et fonctions de la chronique des faits divers, P. : Anthropos, 1970,p. 177).

5. Dans cette forme de représentation ou de mise en scène, on est au grand jour, les victimes sont nommées et limitées, on a recours à l'institution du « Droit » - avec tous les guillemets qui s'imposent évidemment - , c'est le triomphe de la publicité, il y a ici obligation à se prononcer, contrairement à l'imposition au silence de la terreur quotidienne, etc. Voir Véronique Garros, «Voix inouïes sous la terreur: les grands procès revisités dans "l'intimité diariste" (URSS 1936-1938) », in Claude Pennetier et Bernard Pudal, éds., Autobiographies, autocritiques, aveux dans le monde communiste, P. : Belin, 2002, p. 103-115. 
nous de N. Buharin, ex-membre du Politburo, qui susurrait la nuit à l'oreille de son épouse :

Je ne comprends rien, je n'arriverai jamais plus à comprendre, mais que se passe-t-il donc ?

Sans nom, ce journal l'est enfin en raison des mentions ou titres que portent ses trois cahiers. L'année 1936 s'écrit sous forme de «Notes personnelles». L'année 1937, elle, s'écrit désormais comme « Notes et notes fortuites sur le passé ». Si celles-ci répondent bien, initialement, au genre du journal, elles finissent par adopter une autre forme narrative: l'autobiographie. Mutation nullement fortuite, car, à nos yeux, elle reflète un dire obligé de la terreur. Par ailleurs, s'il fallait donner un sous-titre à cette narration de soi élaborée par N., «L'accusé a la parole» conviendrait. En effet, si ces cahiers ne recèlent presque aucune « intimité $»^{7}$, ils s'apparentent à un discours, ou plutôt à un cri de défense du futur accusé, d'un accusé qui ne parlera pas. Gogol ne nous a-t-il pas prévenus : «on écrit ce qu'on ne dit pas tout haut »? Ainsi, à propos de la réunion au cours de laquelle il a été exclu du parti, N. note ce jour-là : " Pour l'essentiel, je me suis tu ». En revanche, dans l'entre-quatre-murs de son cahier personnel, il aura à cœur d'esquisser, de bredouiller, de prononcer son discours de défense... Ce « je me suis tu » nous paraît faire écho à un « vrai dire » de Paul Ricœur :

À vrai dire, omettre et supporter, voire subir, souffrir, sont autant des faits d'interaction que des faits de compréhension subjective. Ces deux termes rappellent qu'au plan de l'interaction comme à celui de la compréhension subjective, le non-agir est encore un agir : négliger, omettre de faire, c'est aussi laisser faire par un autre, parfois de façon criminelle ; quant à supporter, c'est se tenir soi-même, de gré ou de force, sous la puissance d'agir de l'autre ; quelque chose est fait à quelqu'un par quelqu'un ; supporter devient subir, lequel confine à souffrir. En ce point, la théorie de l'action s'étend des hommes agissants aux hommes souffrants. ${ }^{8}$

Des rumeurs et des soupçons à l'injonction d'autocritique, de l'exclusion du parti à l'arrestation - l'objectif n'est pas ici de narrer dans le détail la descente aux enfers que $\mathrm{N}$. a connue, comme d'innombrables milliers de ses concitoyens. Le biais du raccourci peut cependant permettre de mieux camper le personnage (un « homme-

6. Véronique Garros, «L'erreur de Staline », préface à Anna Larina Boukharina, Boukharine, ma passion, P. : Gallimard, 1990 (Au vif du sujet), p. 16.

7. Peut-être au sens que « Bataille assigne au concept d" "intimité" et dont il faut aussitôt ajouter qu'il n'a de sens qu'au prix d'une révision complète du concept d'“individualité" telle qu'il prévaut dans notre tradition philosophique. Car dans l'intimité s'abolit le partage entre l'homme et l'univers, entre le sujet et l'objet. La conscience personnelle se dissout pour être rendue au grand jeu impersonnel du monde», Philippe Forest, La beauté du contresens. Allaphbed I, Nantes : Cécile Defaut, 2005, p. 215.

8. Paul Ricœur, Soi même comme un autre, P. : Seuil, 1990, p. 185-186. 
frontière ») et sa situation, ou plutôt l'évolution de sa situation réelle et narrative où l'on voit l'extime dériver vers l'intime.

Première page du journal, datée du 1er janvier 1936 :

Nouvel An, nouvelles pensées en tête. Malgré tout, le regard se tourne et revient sur l'année écoulée, sur quelques « détails » qui ont une importance énorme, radicale, et engagent à avoir de nouveaux points de vue sur de vieilles choses. Premier détail. À mon retour de congé, le Comité du parti a donné l'instruction «d'apprendre les danses occidentales » et, effectivement, aujourd'hui tout le Comité du parti danse des «fox», des «tangos », des «two-steps », le diable seul sait tous leurs noms. Deuxième détail. Dans la Pravda a paru une lettre de Postyšev sur la nécessité de faire des sapins pour les enfants - à laquelle était adjointe une caricature bienveillante d'enfants reconnaissants - , lettre se plaignant du fait que le commerce de sapins n'est pas organisé. Il y a peu encore, ces deux détails étaient considérés par les membres du parti comme des préjugés non éthiques et bourgeois - et on se faisait chauffer les oreilles pour ça. Les temps changent, et l'éthique du parti change. Troisième « détail »: le décret du CIK [Comité central exécutif] du 29 décembre 1935 sur l'accès aux établissements d'enseignement supérieur et aux écoles techniques. On a abrogé toutes les restrictions liées à l'origine sociale des parents ou à leurs privations de droits civiques. Bien que ce dernier détail soit passé presque inaperçu, il a une importance politique exceptionnelle. Tous ces détails témoignent de l'assise et de la solidité du pouvoir soviétique, du fait qu'effectivement la vie est devenue meilleure et plus gaie. Encore un détail. Pour la première fois, il est autorisé de fêter le Nouvel An. Plus encore, il est interdit de convoquer des réunions de bilan ou de travail ce jour-là. Dansez, réjouissez-vous. Et effectivement, les gens ont fait une fête de tous les diables. C'est bien...

On le voit ici, cette future terreur sans nom est une terreur qui ne s'annonce pas...

Les dernières pages (non datées, mais écrites vraisemblablement en novembre 1937) sont les rares moments où N. évoque la vie quotidienne. Après avoir été exclu du parti, on lui a retiré son appartement de fonction et il décrit la chambre dans laquelle il vit désormais avec sa famille - chambre qu'il qualifie de « magasin d'antiquités » car s'y trouvent, entre autres, un piano, un panier pour le chien, un énorme vase chinois, un fauteuil branlant sur lequel sont juchées valises et lampes, etc. Après avoir évoqué le panier du chien logé sous le piano, il poursuit :

Comique et risible, si ce n'était pas, par ailleurs, tragique. Parce que dans ce « magasin d'antiquités », ou plutôt cet entrepôt, vivent aussi des gens dont la vie entière est également brisée, anéantie - comme après une catastrophe naturelle : un déluge, un incendie ou un tremblement de terre. Ici vit maintenant un ex-tout-puissant de ce monde, terreur de la contre-révolution, mais qui est désormais lui-même exclu du parti et attend qu'on le verse dans la catégorie des contre-révolutionnaires, qu'on vienne le chercher et qu'on l'interne dans la prison intérieure du NKVD ou à la Butyrka. «Un ennemi du peuple pas encore totalement dépecé » - c'est ainsi que le qualifient ceux qui mènent campagne contre lui et qui ont besoin de lui comme victime. Quotidiennement, ces mêmes personnes répandent volontairement des rumeurs à son égard, comme quoi une perquisition a eu lieu chez lui, qu'il a été arrêté, déporté. Il s'agit en fait d'une charge intentionnelle provenant d'imposteurs cherchant à nuire. Au cours des 
six derniers mois, lui, en revanche, a désormais les traits tirés, il a d'une certaine façon vieilli et, surtout, il a désormais des cheveux blancs. Il ne reste plus rien de cet homme qui était gai, affable, le chant toujours aux lèvres. Il est cassé, brisé, comme une vieille charrette autour de laquelle tourne son propriétaire depuis déjà six mois en se demandant - faut-il la faire réparer ou tout simplement la porter à la casse ? -, et depuis le temps il ne se résout toujours pas à faire ni l'un ni l'autre. Et la charrette fait ainsi semblant de servir, elle reste dans la cour où tout, autour d'elle, s'effondre et tombe en ruine, où dans la poussière et la boue s'apprêtent à émerger de nouveaux colosses du génie humain. Et que va-t-il devenir ce combattant pour la révolution, cassé et brisé ? Personne ne le sait et personne ne le dira. Et il attend. Il attend, malgré tout, du parti une certaine clémence - bien qu'il sache parfaitement que son destin n'est pas entre les mains du parti, mais entre celles du NKVD [....].

Procès de désubjectivation ce « il » qui se substitue à « je » ? Le fait - fondé a priori sur une rupture à soi - que $\mathrm{N}$. ne peut désormais s'écrire qu'à la troisième personne du singulier? Ne pourrait-on, au demeurant, inscrire cette substitution dans une histoire plus universelle où :

en un moment d'absence à soi, ce qui est peut-être celui de la plus extrême lucidité, l'être se voit coupé de sa mémoire, de sa formation, de ce qu'il est historiquement : c'est cette coupure qui l'amène à une forme de réflexion, à l'écriture. [...] Un individu quelconque se trouve pris dans une situation anormale, de façon si brutale et traumatique que son rapport habituel au monde, ses repères moraux s'en trouvent brisés. Le témoignage est un double récit : récit des événements, mais aussi et peut-être surtout - même si ce n'est pas affiché explicitement - récit de cette brisure. ${ }^{9}$

Comme pour tout journal personnel, il y aurait de multiples entrées possibles pour aborder et rendre compte des « Notes personnelles et fortuites » de N. L'écriture propre de ces Notes engage cependant à privilégier deux « rectangles fictifs » ${ }^{10}$ : la notion de duplicité - le terme dvurušnik (habituellement traduit par « homme à double face ») est en effet une notion centrale en cette époque de terreur ; la ou les façons dont on tente de se faire une raison de la terreur, mais aussi comment, ce faisant, celle-ci parvient - avec plus ou moins de succès - à imposer certaines formes narratives.

9. Laure Himy-Piéri, « "Peut-être que les enfants poseront des questions..." Réflexion sur l'art et la fiction chez Perec et Bober », in Carole Dornier et Renaud Dulong, éds., Esthétique du témoignage, P. : éd. de la Maison des sciences de l'homme, 2005, p. 192.

10. L'expression est empruntée à Roland Barthes : «Le texte, dans sa masse, est comparable à un ciel, plat et profond à la fois, lisse, sans bords et sans repères ; tel l'augure y découpant du bout de son bâton un rectangle fictif pour y interroger selon certains principes le vol des oiseaux, le commentateur trace le long du texte des zones de lecture, afin d'y observer la migration des sens, l'affleurement des codes, le passage des citations », S/Z, P. : Seuil, 1970, p. 18. 


\section{La duplicité comme temps déstructuré11}

Concernant le processus visant à démasquer l'ennemi, le journal de $\mathrm{N}$. décline une gradation via les épithètes visant à le stigmatiser. Au premier stade, vous êtes un $d v u r u s ̌ n i k$ potentiel. Au deuxième stade, vérification faite, vous devenez un vreditel' avéré. Ce terme, habituellement traduit par «saboteur», signifie à l'origine un «parasite des champs», un «insecte nuisible pour les cultures» - et il est ainsi désigné dans le Recueil des lois et des ordonnances du gouvernement ouvrier-paysan de I'URSS (Sobranie zakonov i rasporjaženij raboče-krest'janskogo pravitel'stva SSSR, 1924-1937) jusqu'en 1932. Ce n'est en fait que deux ans plus tard que le sens du mot change radicalement pour désigner désormais des personnes qui nuisent volontairement à d'autres. Comme Vladimir Papernyj raisonne à ce propos, «cette étrange conduite déviante n'apparaît désormais plus comme le résultat de l'action du monde environnant; elle semble s'engendrer d'elle-même en dépit [c'est moi qui souligne - V. G.] du milieu environnant $»^{12}$. Au dernier stade, celui d'ennemi du peuple, vous êtes un homme mort, tout au moins socialement.

N., avant de mourir socialement puis de mourir tout court, oblige à s'intéresser avant tout au qualificatif de dvurušnik qui «met en langue » une réactivation de formes anciennes au cours de cette histoire désorbitée qu'ont constituée les années 1930. À ce propos, Tamara Kondratieva fait remarquer :

A. M. Seličev fut le premier à suivre l'évolution langagière après la Révolution. Le célèbre linguiste V. Vinogradov contribua également à cette recherche. Dans son Histoire des mots, il fait remarquer que l'expression de l'idéologie révolutionnaire est particulièrement imprégnée de slavon. [...] En examinant son Histoire des mots [...], j'ai pu constater qu'un afflux de mots semi-oubliés du slavon ou du langage argotique populaire a eu lieu dans les années postrévolutionnaires. C'est notamment le cas de mots tristement célèbres du régime. Le mot dvurušnik - l'homme «à double face » qu'on appelait à démasquer dans les années 1930 - provient d'un argot populaire de la Russie du sud et ne figure pas dans les dictionnaires de la « langue vivante $»[\ldots] .{ }^{13}$

On conçoit, après ces remarques, les difficultés de traduction imposées par ce terme. S'il sera possible ultérieurement de se référer au dieu latin bifrons, Janus, il serait pour l'heure saugrenu de le faire correspondre à « notre » dvurušnik, tant disparaît, entre autres, la connotation populaire et péjorative du terme à l'époque. Gardons pour l'instant l'idée d'un individu jouant double jeu, avec tout l'implicite de fourberie, d'imposture, de mensonge et de traitrise qu'elle implique - sans oublier que le phénomène n'est en rien une invention purement soviétique. S'interrogeant sur «les

11. «Un temps déstructuré qu'aucun calendrier ne ponctue [plus] », l'expression, empruntée à Michèle Leclerc-Olive, (« Temporalités biographiques : lignes et nœuds », Temporalistes, 44, septembre 2002, p. 37) correspond ô combien, on va le voir, au « cas N. ».

12. Vladimir Papernyj, Kul'tura « Dva », M. : Novoe literaturnoe obozrenie, 1996, p. 192-193.

13. Gouverner et nourrir : Du pouvoir en Russie ( $X V \mathrm{I}^{\mathrm{e}}-X X^{\mathrm{e}}$ siècles), P. : Les Belles Lettres, 2002, p. 16. 
racines de la surprenante duplicité préfigurant la perfidie encore plus grande qui devait jouer un rôle si monstrueux dans la tradition révolutionnaire », Hannah Arendt poursuit par ailleurs : «C'est la guerre à l'hypocrisie qui transforma la dictature de Robespierre en Règne de la Terreur [...] il s'agissait toujours de découvrir ce qui était dissimulé, d'arracher le masque, de dénoncer l'imposture et la duplicité » ${ }^{14}$.

La première chose essentielle à faire remarquer est que notre auteur partage naïvement avec le pouvoir ce présupposé - qui est en fait une injonction idéologico-morale d'une grande violence - selon lequel l'homme peut, et doit, être un, être «transparent». On est donc loin de «l'individu pluriel » mis en lumière par nos sciences sociales, loin de l'idée, ici empruntée à la psychanalyse, selon laquelle « le peuple initialement opposé comme un tout au tyran, se révèle à la fois divisé et double en chaque sujet singulier. Opprimé oppresseur, enchaîné déchaîné, chacun peut, avec des partenaires différents, vivre simultanément toutes les distributions de rôles dans l'espace social ${ }^{15}$.

N., qui sera réhabilité à titre posthume, n'a de cesse, lorsque soupçons, accusations, injonctions à procéder à son autocritique commencent à pleuvoir, de clamer son innocence, sa fidélité sans faille à la ligne générale, son dévouement éternel au parti et à Stalin. Dans son refus à endosser le rôle que le parti tente de lui donner, il serait ici, si l'on suit la logique qu'Hannah Arendt prête à cette terreur, bien dans la situation ainsi résumée :

«Ou bien vous avez commis ce crime» [que le parti dénonce] «ou bien vous avez été appelé par le Parti à jouer le rôle de criminel » - et dans les deux cas vous êtes objectivement un ennemi du Parti... si vous refusez, vous vous mettez en contradiction avec vous-même et, par cette contradiction, vous retirez tout sens à votre vie. ${ }^{16}$

Le parti, donc, exige une posture particulière, « jouant sur les mots » :

Le maintien de soi, c'est pour la personne la manière telle de se comporter qu'autrui peut compter sur elle. Parce que quelqu'un compte sur moi, je suis comptable de mes actions devant un autre. Le terme de responsabilité réunit les deux significations : compter sur..., être comptable de... Elle les réunit, en y ajoutant l'idée d'une réponse à la question : « Où es-tu ? », posée par l'autre qui me requiert. Cette réponse est : «Me voici ! ». Réponse qui dit le maintien de soi. ${ }^{17}$

Peu à peu, cependant, se superpose à la fidélité jurée et aux cris d'innocence de $\mathrm{N}$. ce qui pourrait s'apparenter de prime abord à un lent délitement du croire, et qui débouchera - paradoxe ? - sur une laborieuse reconquête de son ipséité ${ }^{18}$.

14. Hannah Arendt, Essai sur la révolution, P. : Gallimard, 1967, p. 129, 142, 143.

15. Patrice Loraux, Le tempo de la pensée, P. : Seuil, 1993, 452 p.

16. H. Arendt, Le système totalitaire, P. : Seuil, 1995, 313 p.

17. Ricœur, Soi même comme un autre, p. 195.

18. Ce concept, dû à Paul Ricœur, « permet de le différencier de l'identité, notion plus sociale. L'ipséité est cette continuité temporelle du soi, que chacun éprouve normalement sans rupture, 
S'instaure lentement (sournoisement, pourrait-on dire) « une distance tant intime qu'explicite vis-à-vis du dogme auquel il adhère et qui participe d'une philosophie du "comme si" » 19 .

La distance intime se manifeste avant tout sous la forme d'une contradiction entre son corps et les injonctions de ces Temps, qui exigent jeunesse, optimisme, vitalité, voire immortalité :

Ces derniers temps, je ressens une immense fatigue, celle que pourrait éprouver un vieillard.

Je ressemble désormais à une arba ouzbek dont la musique, et le refrain en particulier, rappellent les notes d'une marche funèbre. Elles sont d'autant plus déplacées en cette époque marquée par le début d'une vie pleine, heureuse et gaie $[\ldots]$.

Et sans jamais prononcer le mot, il songe au suicide et écrit une lettre d'adieu à ses proches.

Est-il possible que j'aie déjà donné tout ce que je pouvais, que je ne sois désormais plus utile à personne? Je suis devenu vieux. J'ai les cheveux tout blancs qui a besoin d'un être comme ça ?

D'un point de vue intime, il se trouve donc en porte-à-faux avec l'époque, dans une dualité, voire duplicité, avec celle-ci...

La distance explicite, elle, se manifeste par un aménagement défensif se traduisant par la figure rhétorique du « non... mais », par un discours double. Il doute, par exemple, de la culpabilité de Lacis (un proche « démasqué ») :

Personnellement je doute que le vieux ait eu de quelconques liens avec des saboteurs ou des espions.

Le paragraphe suivant, lui, énonce :

La situation exige d'agir sans faiblir : fusiller les ennemis, point final, - et il poursuit : «Je suis content que Ežov ait commencé à s'attaquer aux gros poissons qui, sans aucun doute, ont nui à la cause et mené un travail en totale contradiction avec les ordres du camarade Stalin. Ežov prend les choses en profondeur, sans négliger un seul côté. Ce n'est qu'ainsi que l'on pourra se débarrasser des ennemis.

Comme tout cela est dur et injuste. Mais bon. Le parti a toujours raison de toute façon.

Ce «non... mais » a un autre versant, remarquable, appliqué à lui-même et qui aboutit à ce paradoxe difficilement acceptable ou pensable pour nous : il finit par

mais qui devient problématique lorsque la biographie inclut des épisodes traumatisants ». (Renaud Dulong, « Transmettre de corps à corps », in Carole Dornier et Renaud Dulong, éds., Esthétique du témoignage, p. 246.)

19. Marc Augé, Pour quoi vivons-nous ?, P. : Fayard, p. 116. 
accepter la logique ou l'illogique de la Terreur et se définir lui-même comme un dvurušnik objectif :

Je le sais depuis longtemps que, dans cette conjoncture de lutte, où le parti mène un combat sans pitié pour éliminer les derniers éléments trotskistes et boukhariniens, les pires ennemis du peuple qui soient — pas une seule faute que j'aie pu commettre ne doit rester impunie. Cela est égal maintenant que je n'ai jamais été trotskiste. De simples liens personnels [avec eux] sont aujourd'hui considérés comme un crime.

Serais-je une exception? Bien sûr que non. Mais je sais, je comprends et j'accepte cette punition comme méritée.

N. est-t-il un cas singulier ? Il serait légitime d'en douter si l'on met en regard ces « paroles énigmatiques » avec une tentative plus générale d'exfolier un sens à ce type d'allégation :

Tout homme [baignant dans la culture des années 1930-40] sent obscurément, qu'au fond de son âme, se loge une part de saboteur et cela éclaire, dans une certaine mesure, les paroles énigmatiques que K. Alabian a prononcées le jour de l'ouverture du premier Congrès des architectes. «Le plenum du Comité central, at-il dit, a attiré notre attention sur une question réelle et véritablement impérieuse de notre vie politique, celle de la lutte contre les ennemis du peuple $[\ldots]$ d'où $[\ldots]$ la nécessité de développer tous azimuts l'autocritique. » Si le moyen de lutter contre l'ennemi est l'autocritique, alors il est clair que l'ennemi se loge en toi-même..$^{20}$

Outre cette intériorisation personnelle de la notion sociale, idéologico-historique d'ennemi intérieur, il convient sans doute de souligner que la victime en suspens fait en sorte sien le principe de la «culpabilité par association (guilt by association)» exprimant, au sens premier du terme, «l'intérêt personnel du pouvoir : découvrir et retracer les liens des inculpés entre eux pour élargir les réseaux de la prétendue conspiration $»^{21}$.

Cependant, si l'on revient à une logique non terroriste, l'on pourrait accepter le syllogisme de ce paradoxe, mais dans un autre sens: N. est effectivement un dvurušnik, non au sens contemporain d'ennemi non démasqué, mais au sens du dieu Janus, celui de l'entre-deux.

Mais un entre-deux insigne, qui ne parvient plus à faire le passage entre son passé de vieux bolchevik et cette ère nouvelle, ainsi qu'il la désigne en 1936. La Terreur est due in fine à la montée d'une nouvelle génération qui veut se faire une place au soleil et cherche à éliminer les vieux bolcheviks :

Lui [un autre accusé], tout comme moi, sommes de vieux pédagogues. Nous ne convenons plus à ces ordures qui ont relevé la tête et cherchent à détruire les immenses conquêtes de la révolution.

20. Papernyj, Kul'tura «Dva », p. 216-217.

21. Malte Griesse, compte rendu de Reinhard Müller, Menschenfalle Moskau : Exil und stalinistische Verfolgung, Hamburg: Hamburger Edition HIS Verlag, 2001, Cahiers du Monde russe, 43 (4), 2002, p. 743. 
C'était une époque magnifique, l'époque de notre jeunesse, l'époque de la jeunesse du pouvoir soviétique. [À propos de l'année 1918.]

[...] beaucoup d'imbéciles, qui n'ont rien fait pour la révolution, mais qui l'ont utilisée pour leurs misérables buts personnels.

Cet entre-deux - contrairement à Janus dont les deux visages évoquent le présent comme transition du passé au futur - ne peut plus, quant à lui, s'inscrire dans le présent ; il inverse cette temporalité du passé au futur. On passe avec lui du futur de plus en plus évanescent - au passé, parfois aussi du passé au futur, mais le présent ne peut plus s'écrire désormais, il est innommable :

[...] quelque chose s'était effondré en silence. [N.] n'avait plus de lieu, ni de nom qui lui dise la vérité de ses exigences. ${ }^{22}$

\section{La terreur « formelle »}

Cette impossibilité de s'inscrire dans une trame historique - donc dans une histoire collective et une temporalité partagée - qu'impose la terreur se traduit, dès lors, aussi sous une forme narrative : phénomène que l'on ne saurait négliger d'un point de vue historiographique. En effet, au fur et à mesure de cette violente expulsion du temps présent, le journal de N. se transforme peu à peu en Mémoires ou éclats d'autobiographie. Les dates quotidiennes disparaissent ; le dernier cahier prend pour titre, comme on l'a dit, « Notes fortuites sur le passé » :

[...] l'horreur a fait son entrée comme par effraction, suscitant la nécessité inouie d'une parole soudainement autre..$^{23}$

Les temporalités concurrentes entre les deux genres font état de manières concurrentes de dire, d'écrire l'histoire. Le journal, « écriture en action », comme le qualifient certains,

signale une nette aversion pour le temps linéaire, téléologique : la structure cyclique imposée par le calendrier débouche sur une sorte de temps rituel, niant toute idée de fin et susceptible de faire échapper au point final [c'est moi qui souligne - V. G.], mais aussi laisse la porte ouverte à l'accident, à la perte, à l'état de suspension, à l'alternative, à la richesse des possibles. ${ }^{24}$

L'autobiographe, lui, « connaissant le mot de la fin, ou plutôt le point d'arrivée de son entreprise, écrit à contretemps ; le fil d'histoire est prédestiné par l'aboutisse-

22. Michel de Certeau, La culture au pluriel, P. : Christian Bourgois, 1980, p. 19.

23. Forest, La beauté du contresens, p. 149.

24. Almuth Grésillon, Éléments de critique génétique : Lire les manuscrits modernes, P. : PUF, 1994, p. 138. 
ment déjà acquis, ce qui impose une sélection rejetant comme inutiles les ouvertures sans suite, les possibilités qui ne s'accompliront pas $»^{25}$.

Au cœur de ces différences gît la violence subie par N. : c'est en fait lorsqu'il entrevoit avec certitude le point final qu'on lui assigne - l'arrestation, la mort qu'il bascule dans l'écriture autobiographique. Le présent n'étant plus vivable et ne pouvant plus s'écrire, l'auteur se retourne vers le passé, vers son enfance et redevient « je ». Telle est l'issue de cette disjonction des chronotopes, de « la distanciation entre l'individu et son contexte. Il peut s'agir d'un détail infime de la vie domestique, comme d'une rupture entre le moi et le monde historique, l'essentiel est ce sentiment de disjonction, qui induit des chronotopes différents : le système cognitif, les valeurs de l'un, configurent un espace-temps qui n'est plus le même que celui de l'autre. C'est peu de dire que temps personnel et temps héroïque ne coïncident pas : le témoin est séparé de son époque $[\ldots] »^{26}$.

On apprend alors que son désir le plus cher a toujours été d'être médecin, qu'il a fait une requête auprès du tsar pour obtenir, au vu de sa situation sociale, une bourse pour faire ses études, que cette requête lui a été refusée. On apprend également qu'une fois entré dans le mouvement révolutionnaire clandestin, il a échappé par chance à l'arrestation - ordonnée, selon lui, par le tsar en personne. La révolution aboutit à un retournement de situation. Un retournement qui mérite la longue citation qui va suivre : il faut dire qu'il est en charge, à Ekaterinburg, de la garde de celui qu'il appelle «ce détenu portant le titre d'ex-empereur de toutes les Russies, sa Majesté le tsar Nikolaj II Romanov » et craint à cet instant une attaque des Blancs pour le libérer.

À ce propos, il écrit a posteriori, donc :

Le Centre ne m'a pas demandé s'il fallait transférer [ou] détenir Nikolaj II à Ekaterinburg. Le Centre ne m'a pas demandé quelle influence cela aurait sur tout le front de l'Oural. Non, on m'a seulement dit : tu réponds de la vie du tsar. Et c'est tout. Et que pouvais-je répondre si ce n'est l'habituelle expression militaire : « À vos ordres !»

$\mathrm{N}$. craint donc une attaque ennemie et continue de raisonner :

Malgré tout, j'étais persuadé que ce ne seraient pas les troupes blanches du front qui parviendraient à libérer Nikolaj II, mais que le coup viendrait d'éléments contre-révolutionnaires encore en liberté se trouvant dans la ville même. Pour écarter ce risque, tout, bien sûr, dépendait de nous seuls, de notre vigilance, des mesures que nous prendrions à cet effet. C'est pourquoi j'ai décidé personnellement d'aller examiner comment Nikolaj II était détenu.

Il occupait un hôtel particulier, superbe et spacieux, en plein centre-ville. J'étais accompagné [pour vérifier dans quelles conditions était détenue la famille impériale] du commissaire politique en chef du front. De l'extérieur, l'endroit où il était détenu se distinguait seulement par le fait qu'il était entouré d'une haute palissade et qu'à l'intérieur était dressée une palissade du même type. Le chef de la garde nous a accueillis et nous a présenté le registre où figuraient la qualité et le

25. Georges Gusdorf, Lignes de vie I : Les écritures du moi cité in Françoise Simonet-Tenant, Le journal intime, P. : Nathan, 2001, p. 80.

26. Himy-Piéri, «"Peut-être que les enfants poseront des questions...”» p. 197. 
nombre des détenus. Y figuraient donc Nikolaj et toute sa famille : ses enfants et sa femme, Aleksandra Fedorovna. Parmi les personnes se trouvant en détention volontaire auprès de lui, il y avait son médecin personnel, le docteur X. Après nous avoir fait son rapport, le chef de la garde nous a fait faire le tour du lieu de détention. En entrant dans le bâtiment, on remarquait avant tout une mitrailleuse disposée entre les portes menant à l'entrée et auprès de laquelle veillait tranquillement un soldat affecté à ce poste. Passée la porte, il y avait un long couloir séparant de droite et de gauche diverses chambres ; au vu des lits, il s'agissait de celles des filles. Plus loin, dans le coin gauche, se trouvait la chambre destinée à Aleksandra Fedorovna et son fils. À droite, il y avait une salle à manger donnant sur la cour. Là aussi une mitrailleuse était installée.

Nous sommes tout d'abord entrés dans la salle à manger où se trouvaient, autour d'une tasse de thé, Nikolaj en personne et son médecin. Nous nous sommes alors présentés ; ils se sont levés et se sont contentés d'un long salut silencieux. Nous aussi sommes restés silencieux et avons traversé la salle pour nous rendre dans la cour intérieure où Nikolaj II, quotidiennement, faisait sa culture physique en coupant du bois sous la surveillance vigilante d'un détachement de gardes composé de Lettons. De retour dans la salle à manger, j'ai demandé à Nikolaj :

- Alors, détenu, avez-vous à vous plaindre de vos conditions de détention ou quelques requêtes à présenter ?

- Non, je ne peux me plaindre de rien - a répondu Nikolaj d'une voix quelque peu éteinte.

Aussitôt je me suis approché de cet ex- « autocrate de toutes les Russies » - il faisait pâle figure. Un vieil homme aux traits tirés, de grosses poches sous les yeux, une barbe de couleur indéfinissable. Rien de semblable à ce que nous avions l'habitude de voir sur les tableaux le représentant. Et dire qu'il y a peu cette loque dirigeait un peuple de 150 millions de personnes, que tous tremblaient devant cette loque et que l'on priait dans toutes les églises pour lui - le voilà désormais, être insignifiant et pitoyable, aux mains de ce même peuple en révolte ! À cet instant, je n'éprouvais personnellement rien d'autre que du dégoût.

Nous nous apprêtions à sortir de la salle pour visiter les lieux plus avant, lorsque Nikolaj s'est à nouveau adressé à moi :

- Monsieur le Commandant ! J'ai une requête à votre égard : puis-je avoir l'autorisation de couper du bois un peu plus longtemps dans la cour?

- Est-ce à dire qu'on ne vous le permet pas ?

- On me le permet, mais pour une durée très réduite.

Le docteur X. s'est alors levé pour dire que cela serait très souhaitable pour la santé de Nikolaj Aleksandrovič.

- Bien, je ne m'y oppose pas. Si vous le voulez, vous pouvez couper du bois plus longtemps. Je vais en donner l'ordre au chef de la garde.

- Je vous en remercie - a dit Nikolaj, en nous suivant alors que nous nous dirigions vers les autres membres de la famille.

Dans la chambre du coin se trouvait Aleksandra Fedorovna avec son fils, un garçonnet rachitique. En entrant dans la pièce, j'ai dit bonjour. Elle a répondu par un signe de tête à peine visible, tout en faisant une grimace de mépris. La voilà, la vraie furie, qu'il faudrait coller au mur et envoyer dans l'autre monde.

- Détenue, avez-vous quelques plaintes ou requêtes à présenter ? - lui aije demandé.

Elle s'est penchée vers son fils, sans même répondre.

Au moment où je m'apprêtais à ressortir, Nikolaj s'est à nouveau adressé à moi :

- J'ai encore une requête à vous présenter. 
— De quoi s'agit-il ? - ai-je répondu d'un ton désormais mécontent.

- Il fait très lourd dans cette pièce. Ne pourrait-on percer un petit vasistas sur cette fenêtre afin de le laisser ouvert durant la nuit ?

- Accordé également. Seulement que l'on installe des barreaux de fer au vasistas, ai-je ordonné au chef de la garde. C'est tout pour les requêtes, détenu ? $[\ldots]$

- C'est tout, monsieur le Commandant, merci ! - m'a répondu Nikolaj II.

Le soir, en me souvenant du lointain passé, j'ai écrit une lettre à ma mère dans laquelle je lui disais: "Maman chérie, je me suis totalement vengé de Nikolaj II. Il est désormais mon prisonnier. Et dire qu'il y a peu encore, il avait donné l'ordre de m'arrêter. Si cela m'était autorisé, je le fusillerais de mes propres mains. Mais, hélas, je dois répondre de sa vie et l'autoriser à couper du bois dans sa prison. Maman chérie, quelle ironie, l'ex-empereur et tsar de toutes les Russies transformé en bûcheron [...] ».

\section{Conviction versus adhésion}

Après ces lignes sur le passé - et l'impossible écriture du présent - , le futur revient sous une forme compensatrice, dans tous les sens du terme. Et ce sont là, véritablement, les dernières lignes de son journal :

Mais il faut cependant cesser de penser à cela [sous-entendu au passé]. La roue de l'Histoire continue à aller de l'avant. Notre prolétariat, qui a pris le pouvoir et établi sa dictature, écrira lui-même sa véritable histoire, l'histoire de sa lutte titanesque. Trockij et ses comparses ont voulu falsifier l'Histoire et ils ont échoué. Et les autres aussi échoueront, quand bien même ils s'y essaieraient. De toute façon, l'Histoire montrera tout le monde sous son vrai jour.

« La perte du moi et l'écueil d'une dérive jusqu'aux confins de la folie rendent nécessaire pour le mystique le maintien de l'institution comme corps qui préserve $»^{27}$. En dépit de tout, le prolétariat - et non plus le parti - , ne reste-t-il pas pour N. ce corps qui préserve?

Au demeurant, les réflexions sur l'économie du croire développées par Michel de Certeau seraient susceptibles d'entamer davantage ce qui a la qualité heuristique de rester une énigme. Énigme que représente, par exemple, le cas de Buharin qui écrivait à la veille de son arrestation une « Lettre aux futurs dirigeants du Parti » dans laquelle il se défend («Jamais, je n'ai jamais été un traître.») et stigmatise les organes du NKVD (« organisation dégénérée de bureaucrates sans idéaux, moralement déchus mais grassement rémunérés [et qui] se parent de l'autorité passée de la Tchéka à seule fin d'alimenter la méfiance maladive de Stalin (j'ai peur d'en dire plus) [....] »28) puis écrit, lors de sa détention, de stupéfiantes « Lettres de repentir à Koba » (à Stalin, donc) ${ }^{29}$.

27. François Dosse, Michel de Certeau, le marcheur blessé, P. : La Découverte, 2002, 655 p.

28. Boukharina, éd., Boukharine, ma passion, p. 362.

29. Outre 1'article de Jochen Hellbeck cité dans notre bibliographie sélective, on pourra se référer à M. Ja. Gefter, « Le dit de la dignité », in Boukharina, éd., Boukharine, ma passion, p. 367-389. 
L'énigme consisterait, entre autres, à accepter qu'il était lui, dans tous les cas à l'instar de N. Et à admettre que l'homme était pluriel en cette grammaire politique particulière qu'était le stalinisme, comme nous tous, «porteurs d'une pluralité de dispositions, de façons de voir, de sentir et d'agir $»^{30}$.

Dans le cas de N., nous avons laissé en suspens l'idée d'un lent délitement du croire. Mieux vaudrait, pour revenir à Michel de Certeau, parler en termes de hiatus entre conviction et adhésion qui surgit lorsque le discrédit des autorités est tel que ces dernières deviennent insensées « dans la mesure où elles ne correspondent plus à la géographie réelle du sens »; lorsque disparaît un ordre, des raisons de vivre communes.
Alors un exil se produit. [...] Ce qui émigre, avec éclats et protestations quelque- fois, mais plus souvent sans bruit et comme une eau qui fuit, c'est l'adhésion - celle de citoyens, celle d'inscrits à un parti ou à un syndicat, celles de membres d'une Église. L'esprit même qui animait les représentations les quitte. Il n'a pas disparu. Il est ailleurs, parti à l'étranger, loin des structures que son départ change en spectacles désolants ou en liturgies de l'absence. ${ }^{31}$

En ce sens, N. serait bien l'un de ces «apatrides d'une exigence qui n'a plus de représentations sociales », politiques encore moins.

Un espoir reste à formuler : qu'un beau jour le journal de N. devienne accessible à tous et récuse ainsi une implacable sentence :

Sans visage et sans nom, sans image de soi désormais à réfléchir dans aucun miroir, passé par une certaine expérience de la mort, un écrivain est un fantôme qui pourtant s'en revient et qui, coupable et inquiet, poussé par le désir, demande au texte de sa vie le répit passager d'une hospitalité nocturne au terme de laquelle tout - son corps et son œuvre - nécessairement se dissipera au matin. 32

Bref, que notre diariste recouvrant visage et nom cesse enfin d'être un fantôme.

\section{Centre d'étude des mouvements sociaux/Institut Marcel Mauss/EHESS-CNRS}

garros@ehess.fr

30. Voir en particulier le chapitre «Des individus pluriels», Philippe Corcuff, Les nouvelles sociologies : Constructions de la réalité sociale, P. : Nathan, 1995, p. 96-114.

31. Certeau (de), La culture au pluriel, p. 21-24.

32. Forest, La beauté du contresens, p. 310. 